\title{
IDENTIFICAÇÃO DAS EMOÇÕES NO PROCESSO DE CO-DESIGN
}

\author{
Prof $^{a}$. Me. Luciene Machado \\ Universidade do Vale do Rio dos Sinos - UNISINOS \\ luka_mac@hotmail.com \\ Prof. Me. Marcelo Barbosa Corrêa \\ Universidade do Vale do Rio dos Sinos - UNISINOS \\ marcelo@agenciacloser.com.br \\ Prof $^{a}$. Me. Marcia Diehl \\ Universidade do Vale do Rio dos Sinos - UNISINOS \\ marciadiehl@gmail.com \\ Francisco Ximenes \\ Universidade do Vale do Rio dos Sinos - UNISINOS \\ franximenes@ibest.com.br
}

Resumo: O Design é uma área de caráter projetual que utiliza diversos processos de geração de ideias para tangibilizar o conceito de projeto de um produto ou serviço. Os designers podem projetar sozinhos ou junto a outros designers, que podem ser de áreas divergentes. Neste processo, algumas emoções podem estimular ou bloquear a geração de ideias entre os designers, podendo alterar os resultados do produto final a ser projetado. Dentro deste contexto, este artigo mostra os resultados de uma pesquisa exploratória concluída, cujo objetivo foi identificar as emoções envolvidas durante a geração de ideias em processos de co-design entre designers, a fim de propor diretrizes para a criação de um espaço físico modelo que potencialize a geração de ideias. O método utilizado foram entrevistas em profundidade com base nos fundamentos da Appraisal Theory, aplicadas a designers de áreas diversas. Os resultados apontaram que, a partir da identificação das emoções que estimularam o processo de geração de ideias, como o entusiasmo e o divertimento, foram propostos os primeiros indícios de diretrizes projetuais para a criação de um espaço físico modelo, visando estimular as emoções que potencializam a geração de ideias em um processo de co-design.

Palavras-chave: Emoção, geração de ideias, designers, co-design.

Abstract: Design is an area that has a projetual character which uses several idea generation processes to make tangible the concept design of a product or service. The designers can design by their own or with other designers, which can be from divergent areas. In this process, some emotions can stimulate or block the generation of ideas 
among designers and may change the results of the final product to be designed. Within this context, this paper shows the results of a completed exploratory research aimed at identifying the emotions involved during the generation of ideas in co-design processes among designers, in order to propose guidelines for the creation of physical space model that enhance the generation of ideas. The method used was in-depth interviews based on the fundamentals of Appraisal Theory, applied to designers from different areas. The results showed that, from the identification of emotions that stimulated the idea generation process, such as enthusiasm and enjoyment, were proposed early project guidelines to create a physical space model to stimulate the emotions that enhance the idea generation in a co-design process.

Keywords: Emotion, generation of ideas designers, co-design.

\section{INTRODUÇÃO}

Dentre as formas de se realizar uma atividade projetual está o co-design, o qual se refere a um trabalho colaborativo que pode ser apenas entre designers ou entre designers e não-designers (como por exemplo os usuários) para desenvolver o projeto de um produto ou serviço.

Considerando-se a influência do bem-estar dos integrantes neste processo, compreender as emoções que estimulam e bloqueiam a geração de ideias torna-se relevante para os resultados do projeto. Neste sentido, os estudos sobre emoção têm se mostrado cada vez mais alinhados a projetos de design, possibilitando criar artefatos que suscitam respostas emocionais dos usuários para garantir sucesso comercial. O Design para Experiência é uma das abordagens do Design cuja pesquisa pode ser entendida como dimensões emocionais que enriquecem a experiência do usuário, além da forma, função e usabilidade. Uma das abordagens do Design para Experiência é a Appraisal Theory ("Teoria de Avaliação"), que proporciona aos designers identificar as emoções do usuário a partir de um appraisal (avaliação) de determinada situação, produto ou serviço.

Durante o processo de geração de ideias para se criar um novo produto, podem surgir vários tipos de emoção ao se trabalhar com co-design entre designers. Tais emoções podem ser responsáveis por estimular ou bloquear as ideias, interferindo no resultado. Nesse sentido alguns questionamentos foram feitos: quais as emoções que poderiam estimular e/ou bloquear a geração de ideias? Quais seriam os concerns (preocupações e interesses) que proporcionariam o aparecimento de certas emoções durante a geração de ideias? Seria o ambiente, a atividade de co-design, os materiais disponíveis?

Neste contexto, este artigo mostra os resultados de uma pesquisa exploratória concluída, cujo objetivo foi identificar as emoções envolvidas durante a geração de ideias em processos de co-design entre designers, a fim de propor diretrizes para a criação do espaço físico modelo que potencialize a geração de ideias. O método utilizado foram entrevistas em profundidade com base nos fundamentos na Teoria dos Appraisals (Appraisal Theory), aplicadas a designers de áreas diversas.

A seguir, a conceituação de co-design, emoção e Appraisal Theory, seguida do método e resultados.

\section{CO-DESIGN, EMOÇÃO E APPRAISAL THEORY}


Co-design é um termo que deriva da expressão "co-criação de valor", uma abordagem utilizada inicialmente no meio empresarial por Prahalad e Ramaswany (2004). Esta coloca o consumidor como participante do processo de criação do produto/serviço desde o início do processo. A co-criação refere-se a qualquer ato de criatividade coletiva, ou seja, a criatividade que é compartilhada por duas ou mais pessoas. É um termo amplo com aplicações que vão desde o físico ao metafísico e do material para o espiritual.

Para Sanders e Stappers (2008), o co-design é uma instância específica da cocriação, que se refere a uma atividade conjunta em um processo de desenvolvimento de projeto de um produto ou serviço, realizada somente por designers, ou entre designers e não-designers. Rizzo (2009) destaca que o co-design vai além do envolvimento do cliente final, que tradicionalmente acontece por grupos focais, observações e entrevistas, caracterizando-se pela colaboração pró-ativa do cliente durante todo o processo criativo. As emoções geradas pelos designers durante o processo de criação, também podem interferir no desempenho de suas atividades projetuais. Por esta razão, este artigo busca identificar as emoções que estimulam e bloqueiam os designers em um processo de co-design.

As emoções podem ser definidas como reações afetivas de valência à percepção de objetos e situações. Segundo Maturana (2001), consistem em disposições corporais dinâmicas que especificam os domínios de ações nos quais os animais, em geral, e nós seres humanos, em particular, operamos num instante. Consequentemente, todas as ações surgem e são realizadas em algum domínio emocional e é a emoção que define o domínio no qual uma ação acontece. Por este motivo, pretende-se compreender qualquer atividade humana, atentando para a emoção que define o domínio de ações no qual determinada atividade acontece, para, no decorrer do processo, aprender a ver quais ações são desejadas naquela emoção.

Pela abordagem da neurociência (EAGLEMAN, 2011) o cérebro tem dois sistemas: o racional e o emocional. O sistema racional é aquele que se importa com a análise das coisas no mundo, enquanto o emocional monitora o estado interno e se preocupa se as coisas serão boas ou ruins. Em outras palavras, a cognição racional envolve eventos externos, enquanto a emoção envolve seu estado interno. As decisões quanto à prioridade das ações são determinadas por nossos estados internos (emoção).

De acordo com o modelo de processo de componentes de Scherer (2005), uma emoção é um estado afetivo que encarna cinco componentes: avaliação cognitiva, sintomas corporais, tendências de ação, expressão facial e vocal, e consciência subjetiva da emoção (SCHERER, 1982, 2005). A emoção é limitada na sua duração e intencional (direcionada a um determinado objeto). Com base nestas duas características, a emoção difere de outros estados afetivos como humor ou sentimento (FRIJDA, 1994; DESMET, 2002).

O Design para Experiência (JORDAN, 1999; DESMET, 2002; NORMAN, 2004) traz a dimensão emocional da experiência, através da qual se pode identificar os tipos de emoção, de acordo com os interesses e/ou preocupações (denominados de concerns) dos usuários. Para isso, a Appraisal Theory (DEMIR et al., 2009) foi aplicada como base para a identificação de tais emoções. Esta teoria estabelece a emoção como o resultado de uma avaliação feita pelo usuário em relação a um produto ou serviço, associada ao bem-estar. Também propõe entender como a relação de causa é 
estabelecida entre a avaliação e a emoção e como uma avaliação pode ser um gatilho para identificar uma emoção. Tal teoria pode ser associada à área do Design a fim de prover possibilidades de design para potencializar ou evitar certas emoções (TONETTO e COSTA, 2011 ).

O uso da Appraisal Theory (DEMIR et al., 2009; TONETTO e COSTA, 2011) proporciona aos designers identificar as emoções do usuário a respeito de uma determinada situação, produto ou serviço, traduzindo-os em interesses. Esta teoria descreve o processo cognitivo pelo qual os indivíduos avaliam os estímulos do ambiente que são relevantes para o bem-estar e gatilhos emocionais que preparam o corpo para ação (FRIDJA, 1986; LAZARUS, 1991; SCHERER, 1984a). Segundo Tonetto e Costa (2011), seguindo a Appraisal Theory, a compreensão da multidimensionalidade dos appraisals promoverá uma melhor descrição e compreensão das avaliações dos usuários que levam a emoções pretendidas no projeto. Uma compreensão superficial sobre a natureza dos appraisals pode sugerir que eles representam simplesmente os motivos do uso, quando na realidade, apresentam diferentes facetas, desde a consistência do motivo do uso até o potencial de coping percebido em relação a situações adversas.

Um appraisal é definido como uma avaliação rápida de uma situação a respeito do bem estar de alguém. Embora os appraisals sejam automáticos e não-verbais, pode-se pensar em um appraisal como uma resposta a uma questão: "O que a situação significa para o meu bem estar? Se a resposta a esta pergunta for positiva, uma emoção agradável ocorre. Em oposição, se a resposta for negativa, uma emoção negativa é evocada, segundo Demir et al. (2009). O conceito de appraisal tem sido utilizado para se referir ao conteúdo da experiência emocional e para os antecedentes das emoções (FRIJDA, 1993). Frijda (1993) defende que appraisal no primeiro sentido é o que está contido nas informações em auto-relatos e que esta informação é de uso limitado para fazer inferências sobre antecedentes emocionais. Isto é assim porque a experiência emocional pode conter appraisals que fazem parte da resposta emocional ao invés de pertencer a suas causas. Appraisals resultam frequentemente da elaboração da experiência depois de ter começado a ser gerado (FRIJDA, 1993).

Segundo Demir et al. (2009), a relação entre appraisals e emoções, implica em que o entendimento deste fenômeno pode permitir ativar um padrão de avaliação específico que resulta na emoção correspondente. Isso significa que tentativas de projetar para uma emoção específica podem ser facilitadas pelo entendimento do appraisal que elege esta emoção. Já o concern pode ser definido como o interesse e/ou preocupação do usuário em relação à avaliação de determinado produto ou serviço (Figura 1). Pela Appraisal Theory, o produto e o concern a ele relacionado geram um appraisal (uma avaliação). Este appraisal gera uma emoção, a qual pode ser evitada se for negativa, ou estimulada se for positiva, ao contexto estabelecido. A partir da identificação destas emoções e dos concerns, é possível propor diretrizes projetuais para criar produtos ou serviços que estimulem as emoções desejadas pelo designer, projetando-se assim, para a emoção.

Figura 1 - Modelo básico de compreensão das emoções em relação a produtos. 


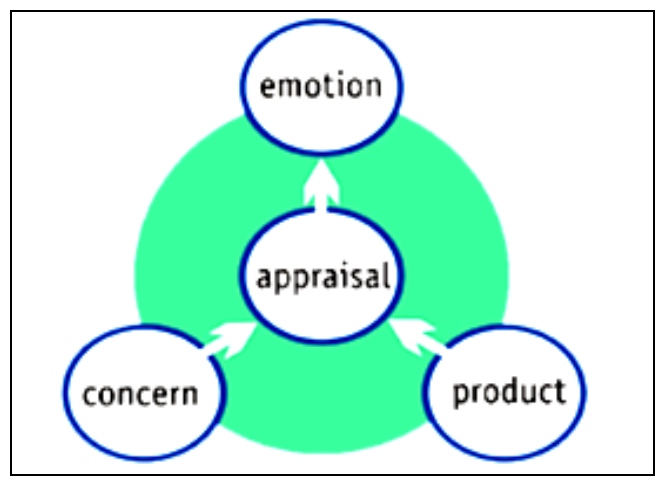

Fonte: Desmet e Hekert, 2007.

Esta abordagem torna-se altamente útil na tarefa de projetar para emoções por possibilitar a compreensão de que a emoção em si não é manipulável, bem como, no caso desta pesquisa, de identificar as emoções dos designers durante uma atividade de co-design. É possível identificar os concerns individuais e entender os appraisals que evocam a emoção específica. Tendo este conhecimento, pode-se por meio da identificação das emoções que estimulam e bloqueiam a geração de ideias em um processo de co-design, propor diretrizes para se criar um espaço físico modelo que potencializa a geração de ideias. A seguir, o método.

\section{MÉTODO}

Esta pesquisa delimitou-se como de natureza exploratória e qualitativa, a fim de identificar as emoções que podem estimular e bloquear a geração de ideias dos designers, durante um processo de co-design. 0 método utilizado foram entrevistas em profundidade com base nos conceitos da Appraisal Theory proposta por Desmet (2002) para identificar os concerns e no "Disco das Emoções" de Scherer (2005) para identificar as emoções. Este disco foi desenvolvido a partir de uma lista de emoções desenvolvida por Scherer (2005), onde um dos lados é verde e mostra as emoções positivas, enquanto o outro é vermelho exibindo emoções negativas, como mostra a Figura 2.

Figura 2 - Os lados do "Disco da Emoções" de Scherer (2005).

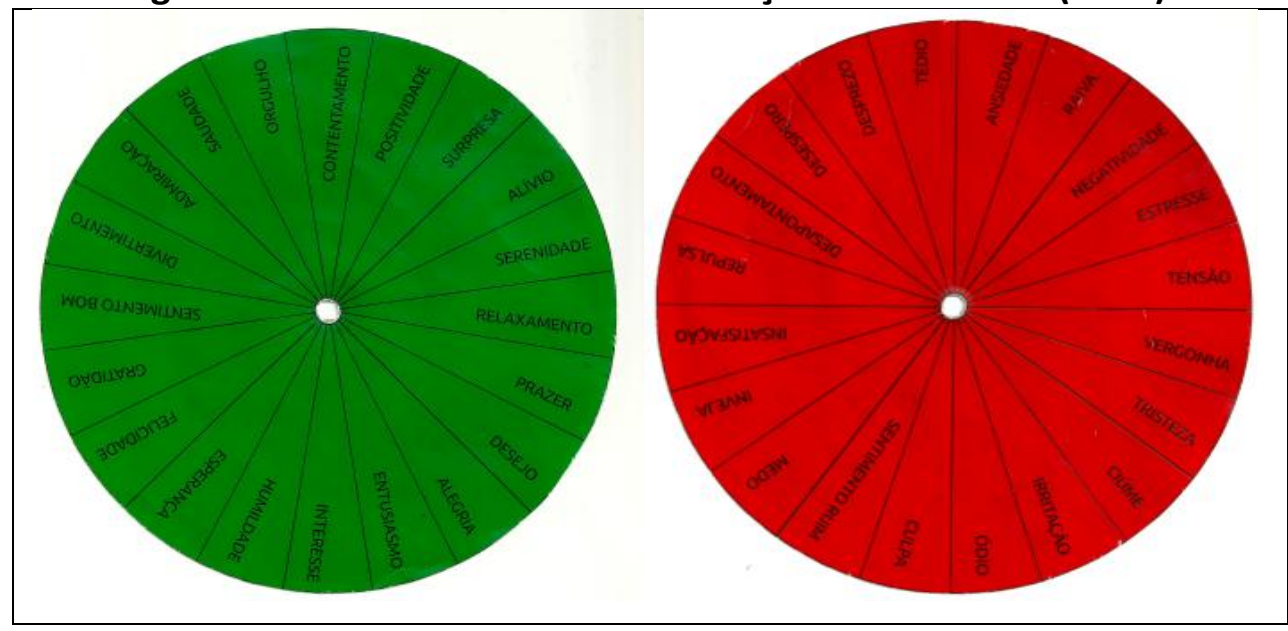

Fonte: Scherer, 2005 
O roteiro semi estruturado abrangia: os fatores responsáveis pela inspiração dos designers na geração de ideias; como é trabalhar em uma atividade de co-design; o processo de criação; as emoções que estimulam e as que bloqueiam o processo de geração de ideias.

Complementando as entrevistas o "Disco das Emoções" foi mostrado aos designers para que identificassem emoções positivas e negativas, relacionadas ao processo de co-design e à geração de ideias. As entrevistas foram aplicadas a designers que já participaram ou trabalham em atividades de co- design. Para determinar o número de designers a serem entrevistados, utilizou-se o critério de saturação, o qual aconteceu depois de 12 entrevistas. Os designers entrevistados eram de diversas áreas, que participam constantemente de atividades de co-design, como: design, design de moda, gráfico, publicidade, arquitetura e design de produto.

As entrevistas foram gravadas em áudio e transcritas. Utilizou-se o método de análise de conteúdo segundo os critérios de Moraes (1999) e Bardin (2011), onde chegou-se aos concerns e às emoções relacionadas à participação em atividades de co-design e ao ambiente físico que tais atividades eram realizadas. A seguir os resultados.

\section{RESULTADOS}

Após as entrevistas, os resultados foram organizados em um Concern Profile, seguindo a abordagem de Demir at al. (2010). Foram identificados dois tipos de concerns dos designers entrevistados: (1) concerns referentes referentes ao espaço físico para a realização das atividades de co-design; (2) concerns referentes à atividade de co-design; bem como, dois tipos de emoções associadas aos mesmos: emoções que estimulam a geração de ideias; emoções que bloqueiam a geração de ideias. A partir da análise do conteúdo das entrevistas, foram identificadas as emoções e os concerns referentes que possibilitaram a construção do Quadro 1 e do Quadro 2. No Quadro 1, estão as emoções que estimulam e potencializam a geração de ideias e os concerns relacionados, identificados pelos designers entrevistados.

Quadro 1 - Emoções que estimulam a geração de ideias e concerns relacionados.

\begin{tabular}{|l|l|}
\hline $\begin{array}{l}\text { Emoções que } \\
\text { estimulam a } \\
\text { geração de ideias. }\end{array}$ & $\begin{array}{l}\text { (1) Concerns referentes ao espaço físico. } \\
\text { (1) Concerns referentes à atividade de co-design. }\end{array}$ \\
\hline Entusiasmo & $\begin{array}{l}\text { (1) "Espaços flexíveis são mais estimulantes. Pode-se molda- } \\
\text { los de acordo com a necessidade". } \\
\text { (2) "O desafio de criar algo é sublime e extraordinário". } \\
\text { (2) "O mais motivador é um projeto que eu vou poder } \\
\text { vivenciar depois". }\end{array}$ \\
\hline Divertimento & $\begin{array}{l}\text { (1) "Ter todos os materiais a disposição: papel, massa de } \\
\text { modelar, brinquedos, bonecos". } \\
\text { (1) "Ter TV, computador, música, DVD, mesa de bilhar, } \\
\text { fliper, e outros para promover momentos de diversão } \\
\text { durante a criação". } \\
\text { (2) "Trabalhar em grupo com pessoas de áreas diferentes". }\end{array}$ \\
\hline Interesse & $\begin{array}{l}\text { (2) "Imersão, concentração em uma única atividade". } \\
\text { (2) "Cada um traz o que tem de melhor da sua área". }\end{array}$ \\
\hline
\end{tabular}




\begin{tabular}{|l|l|}
\hline Serenidade & (1) “O importante também é ter um espaço seu e individual. \\
Sem espaço não se cria, tem que haver a sensação de ter o \\
seu lugar". \\
(1) “Janela grande com vista alta, iluminação não muito \\
clara, prefiro luz amarela". \\
(1) “Estar em um ambiente diurno com luz natural, móveis \\
confortáveis e ergonômicos, sem barulho e com \\
climatização agradável”. \\
(1) “Nos momentos de relaxamento é que os grandes \\
insights vêm".
\end{tabular}

Fonte: Elaborado pelos autores, com base na pesquisa realizada.

No Quadro 2, estão as emoções que bloqueiam a geração de ideias e os concerns relacionados, identificados pelos designers entrevistados.

Quadro 2 - Emoções que bloqueiam a geração de ideias e concerns relacionados.

\begin{tabular}{|l|l|}
\hline $\begin{array}{l}\text { Emoções que } \\
\text { bloqueiam a } \\
\text { geração de ideias }\end{array}$ & $\begin{array}{l}\text { (1) Concerns referentes ao espaço físico. } \\
\text { (2) Concerns referentes à atividade de co-design. }\end{array}$ \\
\hline Tédio & $\begin{array}{l}\text { (1) "Sem internet você não se inspira, não vê o que tá } \\
\text { acontecendo". } \\
\text { (2) "Os projetos que fiz contra vontade, certamente não } \\
\text { tiveram grande inspiração". }\end{array}$ \\
\hline Medo & $\begin{array}{l}\text { (2) “A pressa inibe a inspiração. Fico com medo de não criar } \\
\text { algo inovador". } \\
\text { (2) "O tempo não pode ser nem muito extenso e nem muito } \\
\text { curto, pois pouco tempo é estressante e bloqueia a } \\
\text { inspiração". } \\
\text { (2) “O tempo influencia a inspiração: pouco tempo bloqueia } \\
\text { e muito tempo tende-se a ficar perdido no projeto, dá medo } \\
\text { de perder o foco". }\end{array}$ \\
\hline Insatisfação & $\begin{array}{l}\text { (2) "Ter pessoas totalmente diferentes no grupo pode sair } \\
\text { confusão e não sair projeto algum, não dá certo". } \\
\text { (2) “A partir de seis pessoas não se consegue mais entender } \\
\text { o que elas estão falando. As pessoas se sentem coagidas ao } \\
\text { falar". }\end{array}$ \\
\hline Negatividade & $\begin{array}{l}\text { (2) "Fazer algo que eu não gosto já é desmotivador e } \\
\text { bloqueador da inspiração". }\end{array}$ \\
\hline
\end{tabular}

Fonte: Elaborado pelos autores, com base na pesquisa realizada.

O Concern Profile do Quadro 1 mostra que para se potencializar e estimular a geração de ideias dos designers em uma atividade de co-design faz-se necessário estimular algumas emoções, como: entusiasmo, divertimento, interesse e serenidade, as quais são responsáveis por criar um ambiente de co-design inspirador e propício ao estímulo das ideias. 
O Concern Profile do Quadro 2 apresenta as emoções que devem ser evitadas para não bloquear o processo de geração de ideias, como: tédio, medo, insatisfação e negatividade.

Pode-se constatar que algumas das emoções citadas foram relacionadas com as características específicas da atividade de co-design, incluindo o perfil dos participantes. Outras emoções foram diretamente relacionadas com o espaço físico que deve ser projetado para estimular e potencializar a produção das ideias dos designers.

No caso do entusiasmo, em que o "sublime" e a "vivência do projeto" obtiveram destaque, é possível ver a relação dessa emoção com os fatores internos dos designers. O intangível se faz presente, e permeia a relação do profissional com a prática de criação. O mesmo ocorre com o interesse, onde os valores e características individuais se sobressaem nas citações.

Em relação ao divertimento, as questões emocionais ficaram restritas a fatores externos ao designer, sendo possível observar quais elementos devem compor um espaço físico ideal para a realização de uma atividade de co-design, que estimule a geração de ideias. Para isso, destacaram-se a presença de materiais complementares à atividade, como, TV, computador, massa de modelar, jogos, música, entre outros, para promover o divertimento. Percebe-se, assim, que o bom-humor durante o processo pode ser garantido a partir de materiais físicos que permitam estimular a criatividade.

Quanto à serenidade, o ambiente físico teve relevância fundamental. Para os entrevistados, a sensação de relaxamento durante o processo criativo é derivada das condições ambientais e, consequentemente, sensoriais: mobiliário (conforto e espaço); iluminação; climatização e sonorização. Ficou evidente que é importante para os profissionais a identificação do seu local de criação e trabalho, com características que os aproximem do conforto de suas casas, como disse um dos designers entrevistados: "... tem que haver a sensação de ter o seu lugar".

Em relação às emoções que tendem a bloquear a geração de ideias, o tédio apresentou uma dualidade entre fatores internos e externos aos designers. A obrigação de exercer a tarefa e o limite de acesso às tecnologias devem ser considerados antes do início do processo, a fim de evitar a baixa do nível de inspiração e ideias.

Em relação ao medo, que sempre aparece relacionado ao tempo e à falta deste, foi possível presumir que a limitação temporal é inibidora do relaxamento, servindo como inibidor do desenvolvimento criativo. Embora relevante, essa constatação serve apenas de convite à reflexão, posto que todo processo de co-design tem seu limite de tempo pré-estabelecido para chegar à proposta de soluções.

A insatisfação e a negatividade aparecem estritamente relacionadas ao fator humano. Nos concerns relacionados à insatisfação e à negatividade, os quais são muito próximos ao tédio, encontrou-se a questão do gosto pessoal.

Essa observação permite certos questionamentos, devido ao co-design ser um trabalho colaborativo e com relações interpessoais. A soma de ideias de diferentes pessoas permitiria resultados mais inovadores. Mais precisamente, as respostas apresentaram foco no perfil dos componentes do processo e no número de pessoas. A partir dos resultados coletados, a média ideal de participantes em um grupo seria de até seis integrantes, enquanto que o perfil dos mesmos deveria ser distinto, porém, 
bem definido: liderança, desenvolvedores de ideias, técnicos específicos, julgadores (selecionadores das ideias com maior potencial de êxito) e assim por diante.

\section{CONCLUSÃO}

Esta pesquisa buscou mostrar a aplicação da Appraisal Theory e do Disco das Emoções como facilitadores para identificar as emoções que estimulam e bloqueiam o processo de geração de ideias dos designers em um processo de co-design entre designers, bem como, os referentes concerns desses designers quando participam de uma atividade de co-design.

Verificou-se que dentre as emoções que estimulam o processo de geração de ideias, a que apareceu com maior incidência (o divertimento) foi exatamente o antônimo da emoção que tende a bloquear as ideias com maior incidência (o tédio). Assim, é possível identificar a presença de multidisciplinariedade e de materiais de apoio como suportes estimulantes à produção de ideias. $O$ engajamento no processo e a presença de tecnologias foram identificados como imprescindíveis para evitar o tédio, permitindo que os objetivos do co-design sejam atingidos. Outra questão vem do fato de que todas as emoções que estimulam a geração de ideias estão relacionadas ao movimento, enquanto que a totalidade de emoções que as bloqueiam é referente à inércia, à impossibilidade de continuidade no processo.

A identificação das emoções e concerns relacionados extraídos das entrevistas possibilitou a apresentação dos primeiros indícios de diretrizes projetuais para a criação de um espaço físico modelo, o qual visa estimular as emoções que podem potencializar a geração de ideias em um processo de co-design, como, o entusiasmo e o divertimento:

(1) Quanto aos materiais complementares: identificou-se que é fundamental, além de folhas e papel, a presença de jogos e demais aparatos que estimulem o lúdico dos participantes e promovam interação criativa entre eles.

(2) Quanto às tecnologias: as análises indicaram necessidade de tecnologia em todo o processo, permitindo, assim, uma busca ágil por informações externas e complementares às ideias lançadas durante a atividade projetual.

(3) Quanto ao espaço físico: o espaço ideal deve remeter a um ambiente profissional e, ao mesmo tempo, lembrar o conforto do lar dos participantes. Os elementos para esta finalidade compõem-se de: janelas amplas que permitam boa iluminação e ventilação; isolamento acústico para facilitar a concentração; mobiliário adequado, para promover conforto e relaxamento aos participantes; espaços flexíveis que podem se transformar de acordo com a necessidade.

O intuito dessa proposição de diretrizes foi enriquecer o processo de co-design com a criação de um espaço físico modelo para o desenvolvimento de tal atividade, e assim, garantir maiores estímulos à geração de ideias. Ao pensar em diretrizes, deve-se levar em consideração que é possível planejar e prever o desenvolvimento de algumas condições para se criar um espaço físico modelo para potencializar a geração de ideias, por meio da identificação das emoções e concerns relacionados ao processo de codesign. Observou-se que os fatores internos e subjetivos dos designers participantes não foram passíveis de projetação. Contudo, a identificação desses fatores permitiu minimizar o surgimento de emoções que bloqueariam a geração de ideias e potencializar as emoções que estimulariam a produção de ideias. 
Esta pesquisa não pretendeu esgotar o assunto, sendo que onde o próximo passo é fazer um experimento, utilizando um espaço físico modelo que atenda aos quesitos propostos neste artigo.

\section{REFERÊNCIAS}

BARDIN, Laurence. Análise de conteúdo. Lisboa: Edições 70, 2011.

DEMIR, Erdem; DESMET, Pieter; HEKKERT, Paul. Appraisal Patterns of Emotions in Human-Product Interaction. International Journal of Design, v. 3, n. 2, p. 41-51, 2009.

DESMET, Pieter. Designing emotions. Delft, The Netherlands: Delft University of Technology, Dept. of Industrial Design, 2002.

EAGLEMAN, David. Incógnito: A vida secreta do cérebro. Rio de Janeiro: Editora Rocco Ltda., 2011.

FRIJDA, Nico. H. The emotions. Cambridge, UK: Cambridge University Press, 1986.

The place of appraisal in emotion. Cognition and Emotion, v. 7, n. 3-4, p. 357-387, 1993.

. Varieties of affect: emotions and episodes, moods, and sentiments. In: P.

Ekman; R. J. Davidson (Ed.). The Nature of Emotion, fundamental questions, p. 59-67. Oxford: Oxford University Press, 1994.

JORDAN, Patrick. Pleasure with products: Human factors for body, mind and soul. In: Green W.S.; Jordan P.W. (eds.). Human factors in product design: Current practice and future trends, p. 206-217. London: Taylor \& Francis, 1999.

LAZARUS, Richard S. Emotions and Adaptation. New York: Oxford University Press, 1991.

MATURANA, Humberto. Cognição, Ciência e vida Cotidiana. Belo Horizonte: Editora UFMG, 2001.

MORAES, Roque. Análise de conteúdo. Revista Educação, v. 22, n. 37, p. 7-32, 1999.

NORMAN, Donald. Emotional design: Why we love (or hate) everyday things. New York: Basic Books, 2004.

PRAHALAD, C.K. ; RAMASWAMY, V. O Futuro da Competição. Rio de Janeiro: Elsevier Editora Ltda., 2004.

RIZZO, Francesca. Strategie di co-design: Teoria, Metodi e Strumenti per Progettare con gli Utenti. Milano: Franco Angeli, 2009.

SANDERS, Elizabeth. B. N.; STAPPERS, Peter Jan. Co-creation and the ne landscapes of design. CoDesign: International Journal of Co-Creation in Design and the Arts, v. 4, n. 1, p. 5-18, 2008.

SCHERER, Klaus R. Emotion as a process: Function, origin and regulation. Social Science Information, v. 21, n. 4/5, p. 555-570, 1982.

On the nature and function of emotion: A component process approach. In:

K. R. Scherer \& P. Ekman (Eds.) Approachs to Emotion. Hilldale, NJ: Erlbaum, 1984.

SCHERER, Klaus R. What are emotions? And how can they be measured?. Social Science Information, v. 44, n. 4, p. 695-729, 2005.

TONETTO, Leandro. Miletto. e COSTA, Filipe Campelo da Xavier. Design emocional: conceitos, abordagens e perspectivas de pesquisa. Strategic Design Research Journal, v. 4, n. 3, p. 132-140, 2011. 